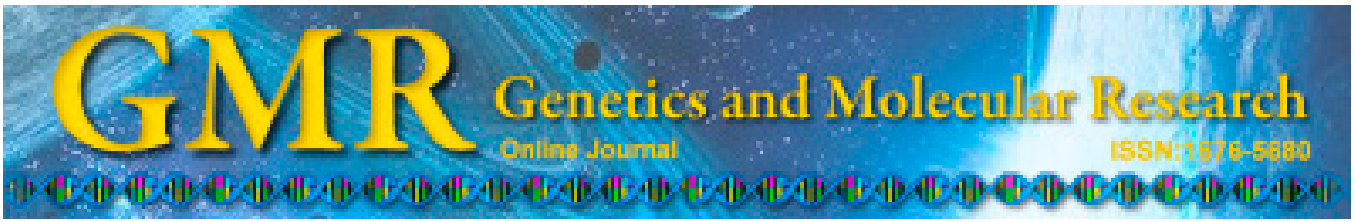

\title{
Genetic polymorphism at the KIR gene locus: determination of gene, genotype, and haplotype frequencies in the Xinjiang Han population
}

\author{
G.-Y. Lin ${ }^{1}$, B. Yu ${ }^{2}$ W.-J. Hu${ }^{1}$, Y.-Z. Zhang1 ${ }^{1}$ X.-J. Zuo ${ }^{1}$ and Y.-B. Wang ${ }^{1}$ \\ ${ }^{1}$ Department of Blood Transfusion, No. 474 People's Liberation Army Hospital, \\ Urumqi, China \\ ${ }^{2}$ First Affiliated Hospital of Xinjiang Medical University, \\ Pacing Electrophysiology Section, Urumqi, Xinjiang, China \\ Corresponding author: B. Yu \\ E-mail: xjlgy_474@163.com
}

Genet. Mol. Res. 14 (2): 6213-6222 (2015)

Received August 20, 2014

Accepted November 13, 2014

Published June 9, 2015

DOI http://dx.doi.org/10.4238/2015.June.9.7

\begin{abstract}
The aim of this study was to explore the genetic polymorphism, genotype, and haplotype characteristics of the KIR locus in the Xinjiang Han population in order to establish a foundation for future analysis of the relationship between KIR genes and disease. KIR genes were detected by sequence-specific primer-polymerase chain reaction in 184 randomly selected, healthy individuals from the Han population in Xinjiang, China. Standard genotype and haplotype analyses were conducted using Hsu's standards classified for analysis. Sixteen KIR genes were detected: $3 D L 3,2 D L 4,3 D L 2$, and $3 D L 2(100 \%) ; 2 D L 1$ and $2 D P 1(99.46 \%) ; 2 D L 3(98.91 \%)$; and so on. The $2 D S 2$ gene frequency was the lowest at $21.74 \%$. Twenty-one genotypes were detected: AJ (2, 2) was relatively common $(42.39 \%)$, followed by $\mathrm{AH}(5,2), \mathrm{AE}(2,8)$ and $\mathrm{H}(2,4)$, with frequencies of $17.39,11.96$, and $8.15 \%$, respectively. In addition, six novel genotypes were identified in 11 Han individuals as well as in other populations in China, which could not be classified for analysis. These results indicated that the Xinjiang Han population shares KIR gene,
\end{abstract}


genotype, and haplotype frequency distributions with the Chinese Han population, but also has unique genotypes and haplotypes.

Key words: Killer cell immunoglobulin-like receptor (KIR); Polymerase chain reaction-sequence specific primers; Genetic polymorphism; Genotype; Haplotype

\section{INTRODUCTION}

Killer cell immunoglobulin-like receptors (KIRs) are a group of specifically recognized human major histocompatibility antigen (MHC-I) molecule receptors expressed on the surface of natural killer (NK) cells and T cells. KIR has been shown to be the next most highly diverse immune regulatory gene family after the discovery of the human leukocyte antigen (HLA) cluster, and KIR receptors have important immunomodulatory effects on NK and T cells. The KIR-encoding locus is on chromosome 19q13.4, and the family group includes 18 genes, namely KIR1D, KIR2DL1-5, KIR2DS1-5, KIR3DL1-3, KIR3DS1, Xv, X, and KIR2DP1. $X v, X$, and KIR2DP1 are pseudogenes, and KIR2DL4, KIR3DL2, and KIR3DL3 are framework genes (Zhang et al., 2003a). Studies have found that the KIR genes are genetically polymorphic and that there is a richly diverse KIR receptor library in different individuals that are expressed in different NK cell clones. The NK cell KIR expression pattern directly regulates their ability to clear foreign and harmful substances, and the KIR genotype of an individual is the most critical factor to determine its expression profile, which plays an important role in the body's natural and acquired immunities. Therefore, KIR genotyping assists with the analysis of its function in certain diseases (Zhang et al., 2003b). Previous studies (Khakoo and Carrington, 2006) have shown that KIR locus polymorphism is associated with certain infectious and autoimmune diseases, cancer susceptibility, and pregnancy. The regulatory effects of KIR in hematopoietic stem cell transplantation have also been of great concern in the clinical field. This study focused on the multi-ethnic region of Xinjiang to determine the distribution characteristics of genes within the polymorphic KIR locus in the Xinjiang Han population. We utilized the sequence-specific primer polymerase chain reaction (SSP-PCR) to perform KIR genotyping and genotype and haplotype analysis of 184 cases of Han volunteers from Xinjiang. The results from this study will lay the foundation for further studies of the association between the KIR gene polymorphism and certain diseases in different groups of people.

\section{MATERIAL AND METHODS}

\section{Study design}

This study was a prospective analysis. DNA was analyzed in vitro using molecular biology detection methods. Study participants consisted of unrelated individuals recruited at random from the Xinjiang Han population. Our study was carried out from November 2009 to September 2011, and analyses were performed in the kidney transplant tissue typing room of the blood purification center in No. 474 People's Liberation Army Hospital.

\section{General information}

Study subjects consisted of kidney transplant HLA-matching donors and recipients 
processed through the No. 474 People's Liberation Army Hospital. All subjects gave their written informed consent. A 4-mL venous whole blood sample was collected from donors and recipients and kept in ethylenediaminetetraacetic acid anticoagulant. Genomic DNA was obtained from 84 unrelated Han individuals from the Xinjiang region. DNA extraction was in strict accordance with the instructions of the PROTRANS DNA rapid extraction kit (Beijing San Taihua Biotechnology Company, Beijing, China). The $\mathrm{A}_{260} / \mathrm{A}_{280}$ ratio of the extracted DNA was from 1.8 to 2.0. The genomic DNA samples were stored at $-20^{\circ} \mathrm{C}$ for subsequent analysis.

\section{Reagents and instruments}

The KIR SSP-PCR kit was provided by Tianjin Xiu Peng Biotechnology Development Co., Ltd. (Tianjin, China). The internal reference control was the conservative fragment of the human growth hormone gene, and the amplification product of the internal reference was 588 bp Taq DNA polymerase (Promega, Shanghai China). The DNA extraction kit was the German PROTRANS DNA extraction kit (Beijing San Taihua Biotechnology Company). The PCR amplification instrument was from BIO-RAD Laboratories (MyCycler ${ }^{\mathrm{TM}}$ Thermal Cycler, USA). The electrophoresis instruments such as the DDY-BC and WD-9413B gel imaging analyzers were supplied by the Beijing Liuyi Instrument Company (Beijing, China).

\section{Detection and genotyping of $K I R$ genes}

The SSP-PCR genotyping method for KIR genes was adopted in all samples collected. KIR2DL1-5, KIR2DS1-5, KIR3DL1-3, KIR3DS1, and the two pseudogenes KIR2DP1 (aka KIRZ) and KIR3DPI (divided into KIRX and KIRXv) were detected from the KIR genetic locus. SSP-PCR amplification primers were designed based on the KIR gene sequence and genotyping literature design strategies, to provide a reference for these strategies or the program used for primer development, and a total of 32 pairs were synthesized by Tianjin Xiu Peng Super Biotechnology Co., Ltd. The human KIR genotype kit primer (locus and size) is shown in Table 1. Amplifications were performed in the BIO-RAD PCR amplification instrument. The PCR volume was $10 \mu \mathrm{L}$ per well, and there were 16 wells per subject. The system contained $160 \mu \mathrm{L}$ dNTP buffer, $20 \mu \mathrm{L}$ genomic DNA, and $1.2 \mu \mathrm{L} 5 \mathrm{U} / \mu \mathrm{L}$ Taq DNA polymerase. The reaction conditions were as follows: $96^{\circ} \mathrm{C}$ for $2 \mathrm{~min}, 96^{\circ} \mathrm{C}$ for $20 \mathrm{~s}$ and $68^{\circ} \mathrm{C}$ for $60 \mathrm{~s}$, for a total of 5 cycles; $96^{\circ} \mathrm{C}$ for $20 \mathrm{~s}, 64^{\circ} \mathrm{C}$ for $50 \mathrm{~s}$, and $72^{\circ} \mathrm{C}$ for $45 \mathrm{~s}$, for a total of 10 cycles; and $96^{\circ} \mathrm{C}$ for $20 \mathrm{~s}, 61^{\circ} \mathrm{C}$ for $50 \mathrm{~s}$, and $72^{\circ} \mathrm{C}$ for $45 \mathrm{~s}$, for a total of 18 cycles. Final extension was at $72^{\circ} \mathrm{C}$ for $5 \mathrm{~min}$. The PCR products were loaded on a $2.5 \%$ agarose gel. Electrophoresis was performed at $160 \mathrm{~V}$ for $12 \mathrm{~min}$, and bands were visualized using the WD-9413B ultraviolet gel imaging analyzer.

\section{Genotype and haplotype analysis}

We adopted the 36 KIR genotypes A to AJ, first defined in Caucasians by Hsu et 
al. (2002). Genotypes and the designated 23 common haplotypes were determined using standard typing methods. The KIR haplotype combinations were projected according to the genotypes. When the same genotype had 2 potential haplotype combinations, it was calculated according to the common haplotype literature (Hsu et al., 2002). Of the 23 common haplotypes in the literature, haplotype 1 and haplotype 2 were type A, and the other 21 haplotypes were type B. The comprehensive classification criteria were that all the haplotypes contained $3 D L 3,2 D L 4$, and $3 D L 2$, and one haplotype contained $2 D L 2$ or $2 D L 3$. One haplotype contained $3 D P 1$ or $3 D P 1 v$. Haplotype B carried one or more of the genes KIR2DL5, KIR2DS1, KIR2DS2, KIR2DS3, KIR2DS5, and KIR3DS1, but haplotype A did not include these genes.

Table 1. Human KIR genotype kit primers [locus and size (in bp)].

\begin{tabular}{|c|c|c|c|c|c|c|c|}
\hline $\mathrm{H}$ & G & $\mathrm{F}$ & E & $\mathrm{D}$ & $\mathrm{C}$ & B & A \\
\hline DL1, 145 bp & 2DL2, $146 \mathrm{bp}$ & $2 \mathrm{DL} 3,153 \mathrm{~b}$ & 2DL4, 135 & 2DL5, 145 & 2DS1, 96 bp & 2DS2, $140 \mathrm{bp}$ & $2 \mathrm{DS} 3,158 \mathrm{bp}$ \\
\hline 2DS4, $133 \mathrm{bp}$ & 2DS5, $110 \mathrm{bp}$ & 3DL1, $112 \mathrm{bp}$ & 3DL2, $135 \mathrm{bp}$ & 3DL3, $196 \mathrm{bp}$ & $3 \mathrm{DS} 1,109 \mathrm{bp}$ & $2 \mathrm{DP} 1,168 \mathrm{bp}$ & 3DP1, $131 \mathrm{bp}$ \\
\hline
\end{tabular}

\section{Statistical analysis}

According to the literature (Hsu et al., 2002), the KIR gene detection frequency (F) was measured by counting: $\mathrm{F}(\%)=$ the positive gene number/the total number in the research group. The KIR gene frequency was calculated as follows: GF $=1-(1-\mathrm{f})^{1 / 2}$; genotype frequency was the genotype positive number $n_{1} / \mathrm{N}$; the haplotype frequency was the single haplotype positive number $\mathrm{n}_{2} / 2 \mathrm{~N}$, where $\mathrm{N}$ is the number of study individuals.

\section{RESULTS}

\section{KIR gene frequency distribution}

The $3 D L 3,2 D L 4,3 D P 1$, and $3 D L 2$ genes existed in all individuals from the Xinjiang Han population, yielding a gene frequency of $1(100 \%) .2 D L 1,2 D P 1$, and $2 D L 3$ were common, with gene frequencies of $99.46,99.46$, and $98.91 \%$, respectively; followed by $3 D L 1$ and $2 D S 4$, with frequencies of 91.30 and $89.13 \%$, respectively. The $2 D S 2$ gene frequency was the lowest at $21.74 \%$ (Table 2).

\section{KIR genotype frequencies}

There were 21 genotypes detected in the Xinjiang Han population. The AJ $(2,2)$ genotype was relatively common, with a frequency of $42.39 \%$, followed by AH $(5,2)$, $\mathrm{AE}(2,8)$, and $\mathrm{H}(2,4)$, with frequencies of $17.39,11.96$, and $8.15 \%$, respectively. The $\mathrm{R}(6,9), \mathrm{AA}(4,4), \mathrm{AF}(1,2)$, and $\mathrm{T}(8,8)$ genotypes showed the lowest frequencies, at 
$0.54 \%$. Of 21 genotypes, 15 were the same as in the 36 genotypes assigned by Hsu et al. (2002). In addition, six new genotypes were found in 11 individuals who could not be classified according to the related literature (labeled HXJ1-6) and thus for which genotype analysis could not be performed based on existing standards. The frequencies for these were low, $2.72,1.09$, and $0.54 \%$, respectively. In contrast, the $\mathrm{J}(1,21), \mathrm{B}(3,6)$, $\mathrm{R}(6,9)$, and AA $(4,4)$ genotypes identified in five individuals had not been reported in the Han population in current literature; these exhibited frequencies of $1.09,0.54,0.54$, and $0.54 \%$ (Table 3 ).

\section{KIR gene haplotype frequencies}

Nine haplotypes were detected in 184 individuals from the Han population; haplotypes $1,2,3,4,5,7,8,17,21$. The most common haplotype, $2(\mathrm{~N}=212)$, was found in $61.3 \%(212 / 346)$ of individuals, followed by haplotype $5(\mathrm{~N}=44)$, which accounted for $12.7 \%(44 / 346)$. Haplotype $1(\mathrm{~N}=29)$ accounted for $8.4 \%(29 / 346)$ and haplotype $6(\mathrm{~N}$ $=23$ ) accounted for $6.7 \%(23 / 346)$. Four haplotypes accounted for $89.0 \%$. Haplotypes 7 , $10,11,12,13,14,15,16,18,19,20,22$, and 23 were not detected in this study. Haplotypes 1 and 2 belonged to haplotype group A. Eighty-two cases were haplotype A with a frequency of $69.1 \%$, and the ratio of haplotypes A and B was 2.23:1. In addition, six new genotypes were found, for which haplotype analysis could not be performed based on Hsu's standards (Hsu et al., 2002), suggesting that these may be new haplotypes (Table 4).

$K I R$ gene frequencies in the Xinjiang Han population were comparable with other populations, as shown in Table 5 .

\section{Table 2. Distribution of $K I R$ gene frequencies in the Xinjiang Han population $(\mathrm{N}=184)$.}

\begin{tabular}{lccc}
\hline KIR gene & $\mathrm{N}$ & $\mathrm{F}(\%)$ & GF \\
\hline 3DL3 & 184 & 100.0 & 1 \\
2DS2 & 40 & 21.74 & 0.1154 \\
2DL2 & 41 & 22.28 & 0.1184 \\
2DL3 & 182 & 98.91 & 0.8956 \\
2DP1 & 183 & 99.46 & 0.9265 \\
2DL1 & 183 & 99.46 & 0.9265 \\
3DP1 & 184 & 100.0 & 1 \\
2DL4 & 184 & 100.0 & 1 \\
3DL1 & 168 & 91.30 & 0.7050 \\
3DS1 & 81 & 44.02 & 0.2518 \\
2DL5 & 89 & 48.37 & 0.2815 \\
2DS3 & 44 & 23.91 & 0.1277 \\
2DS5 & 59 & 32.07 & 0.1758 \\
2DS1 & 84 & 45.65 & 0.2628 \\
2DS4 & 164 & 89.13 & 0.6703 \\
3DL2 & 184 & 100.0 & 1 \\
\hline
\end{tabular}

$\mathrm{F}=$ gene detection rate; $\mathrm{GF}=$ gene frequency. 


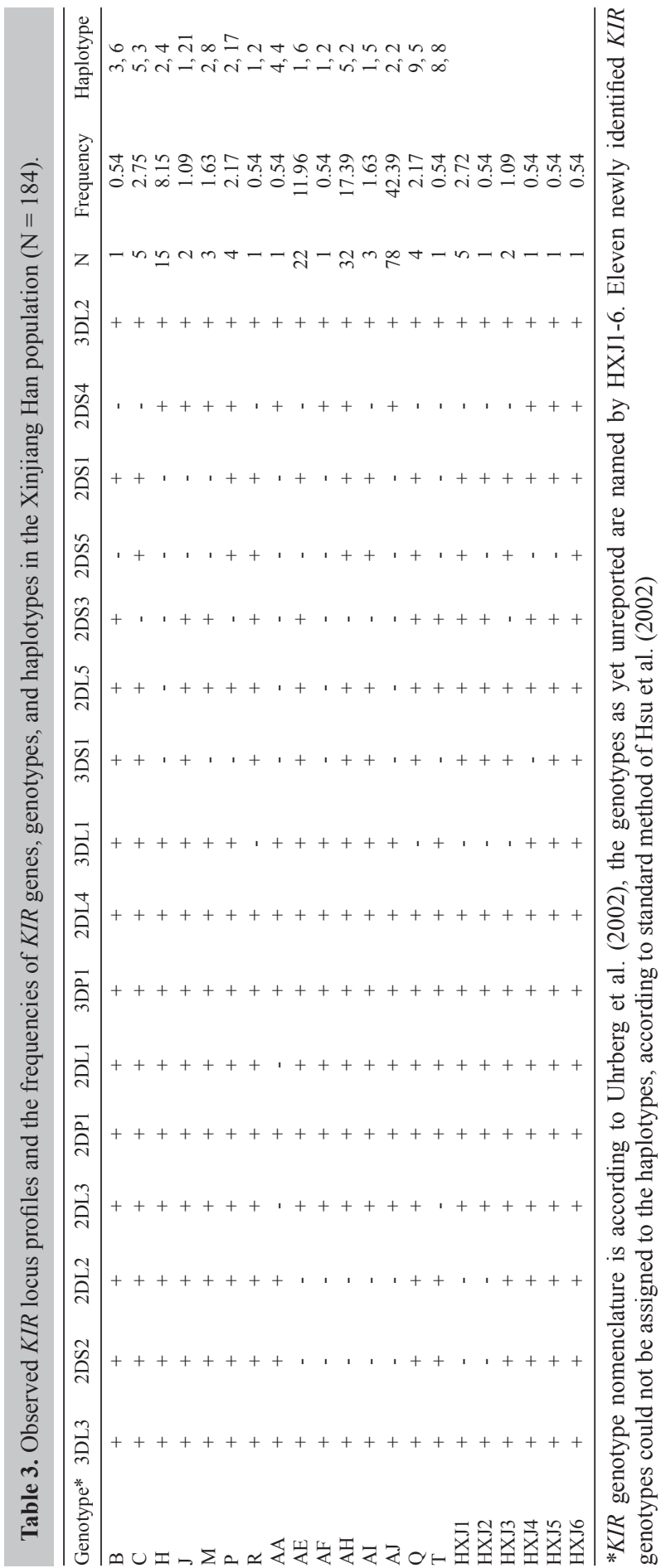


Table 4. Haplotype profiles and frequencies in the Xinjiang Han population $(\mathrm{N}=173)$.

\begin{tabular}{|c|c|c|c|c|c|c|c|c|c|c|c|c|c|c|c|c|c|c|c|}
\hline $\mathrm{Ha}$ & otype & 3DL3 & 2DS2 & 2DL2 & 2DL3 & 2DP1 & 2DL1 & 3DP1 & 2DL4 & 3DL1 & 3DS1 & 2DL5 & $2 \mathrm{DS} 3$ & 2DS5 & 2DS1 & 2DS4 & 3DL2 & $\mathrm{N}$ & $F(\%)$ \\
\hline \multirow[t]{11}{*}{$\overline{\mathrm{A}}$} & 1 & + & - & - & + & + & + & + & + & + & - & - & - & - & - & - & + & 29 & 8.4 \\
\hline & 2 & + & - & - & + & + & + & + & + & + & - & - & - & - & - & + & + & 212 & 61.3 \\
\hline & 3 & + & + & + & - & - & - & - & + & + & - & - & - & - & - & - & + & 6 & 1.7 \\
\hline & 4 & + & + & + & - & - & - & - & + & + & - & - & - & - & - & + & + & 17 & 4.9 \\
\hline & 5 & + & - & - & + & + & + & + & + & - & + & + & - & + & + & - & + & 44 & 12.7 \\
\hline & 6 & + & - & - & + & + & + & + & + & - & + & + & + & - & + & - & + & 23 & 6.7 \\
\hline & 7 & + & - & - & + & + & + & + & + & - & + & + & + & - & - & - & + & 0 & 0.0 \\
\hline & 8 & + & + & + & - & + & + & + & + & + & - & + & + & - & - & - & + & 5 & 1.5 \\
\hline & 9 & + & + & + & - & + & + & + & + & - & + & + & + & - & + & - & + & 4 & 1.2 \\
\hline & 10 & + & + & + & - & - & - & - & + & - & + & + & + & - & - & - & + & 0 & 0.0 \\
\hline & 11 & + & - & - & - & + & - & - & + & - & - & - & - & - & + & - & + & 0 & 0.0 \\
\hline \multirow[t]{12}{*}{ B } & 12 & + & - & - & + & + & + & + & + & - & + & - & + & - & - & + & + & 0 & 0.0 \\
\hline & 13 & + & + & + & - & - & - & - & + & + & - & + & - & + & + & - & + & 0 & 0.0 \\
\hline & 14 & + & + & + & - & - & - & - & + & - & + & + & - & + & + & - & + & 0 & 0.0 \\
\hline & 15 & + & + & + & - & + & + & + & + & + & - & - & - & - & - & - & + & 0 & 0.0 \\
\hline & 16 & + & + & + & - & + & + & + & + & + & - & - & - & - & - & + & + & 0 & 0.0 \\
\hline & 17 & + & + & + & - & + & + & + & + & + & - & + & - & + & + & - & + & 4 & 1.2 \\
\hline & 18 & + & + & + & - & + & + & + & + & + & - & + & + & - & + & - & + & 0 & 0.0 \\
\hline & 19 & + & + & + & - & + & + & + & + & + & - & + & + & - & - & + & + & 0 & 0.0 \\
\hline & 20 & + & + & + & - & + & + & + & + & - & + & + & + & - & - & - & + & 0 & 0.0 \\
\hline & 21 & + & + & + & - & + & + & + & + & - & + & + & + & - & - & + & + & 2 & 0.6 \\
\hline & 22 & + & + & + & - & + & + & + & + & - & +- & + & - & + & + & - & + & 0 & 0.0 \\
\hline & 23 & + & - & - & - & + & - & + & + & + & - & + & + & - & - & - & + & 0 & 0.0 \\
\hline
\end{tabular}

*Eleven (5.98\%) newly identified KIR genotypes in the 184 samples could not be assigned to the haplotypes according to the standard method of Hsu et al. (2002). F = frequency.

Table 5. Distribution of KIR gene frequencies in the Xinjiang Han population and others (\%) (Su et al., 2008).

\begin{tabular}{|c|c|c|c|c|c|c|c|c|c|c|c|c|c|c|c|c|c|}
\hline Different ethnic groups & $\mathrm{N}$ & 3DL3 & 2DS2 & 2DL2 & 2DL3 & 2DP1 & 2DL1 & 3DP1 & 2DL4 & 3DL1 & $3 \mathrm{DS} 1$ & 2DL5 & $2 \mathrm{DS} 3$ & 2DS5 & 2DS1 & 2DS4 & 3DL2 \\
\hline njiang Han & 184 & 100.0 & 21.7 & 22.3 & 98.9 & 99.5 & 99.5 & 100.0 & 100.0 & 91.3 & 44.0 & 48.4 & 23.9 & 32.1 & 45.7 & 89.1 & 100.0 \\
\hline $\begin{array}{l}\text { Jiangsu Han } \\
\text { (He et al., 2009) }\end{array}$ & 69 & 100.0 & 19.3 & 17.8 & 99.3 & 99.3 & 96.3 & 100.0 & 100.0 & 96.3 & 36.8 & 39.4 & 13.8 & 28.3 & 36.8 & 95.9 & 100.0 \\
\hline $\begin{array}{l}\text { Sichuan Han } \\
\text { (Wang et al., 2010) }\end{array}$ & 135 & 100.0 & 28.2 & 28.2 & 97.8 & 99.3 & 99.3 & 100.0 & 100.0 & 97.0 & 35.6 & 43.0 & 17.8 & 28.2 & 37.0 & 97.0 & 100.0 \\
\hline $\begin{array}{l}\text { Shanghai Han } \\
\text { (Zhang et al., 2003b) }\end{array}$ & 87 & 100.0 & 18.4 & 18.4 & 97.7 & 97.7 & 97.7 & 100.0 & 100.0 & 100.0 & 34.5 & 37.9 & 14.9 & 24.1 & 35.6 & 98.8 & 100.0 \\
\hline $\begin{array}{l}\text { Zhejiang Han } \\
\text { (Zhu et al., 2005) }\end{array}$ & 104 & 100.0 & 17.3 & 17.3 & 99.0 & 99.0 & 99.0 & 100.0 & 100.0 & 94.2 & 32.7 & 35.6 & 12.5 & 23.1 & 33.6 & 94.2 & 100.0 \\
\hline $\begin{array}{l}\text { Yunnan Han } \\
\text { (Su et al., 2008) }\end{array}$ & 150 & 100.0 & 25.3 & 25.3 & 99.3 & 99.3 & 99.3 & 100.0 & 100.0 & 95.3 & 32.0 & 36.7 & 15.3 & 26.7 & 36.0 & 95.3 & 100.0 \\
\hline Singapore Chinese & 46 & 100.0 & 28.3 & 28.2 & 100.0 & 100.0 & 100.0 & 100.0 & 100.0 & 97.8 & 30.4 & 39.1 & 17.4 & 21.7 & 28.3 & 97.8 & 100.0 \\
\hline Hong Kong, China & 100 & 100.0 & 28.0 & 28.0 & 98.0 & 99.0 & 99.0 & 100.0 & 100.0 & 94.0 & 39.0 & 45.0 & 25.0 & 26.0 & 40.0 & 94.0 & 100.0 \\
\hline Brazil & 70 & 100.0 & 53.3 & 52.2 & 94.4 & 96.7 & 96.7 & 100.0 & 100.0 & 95.6 & 41.1 & 58.9 & 38.9 & 32.2 & 37.8 & 95.6 & 100.0 \\
\hline Brazil & 154 & 100.0 & 16.9 & 14.3 & 99.4 & 100.0 & 99.4 & 100.0 & 100.0 & 94.2 & 36.4 & 38.3 & 16.2 & 26.2 & 37.7 & 94.2 & 100.0 \\
\hline Japan & 41 & 100.0 & 14.6 & 14.6 & 100.0 & 100.0 & 100.0 & 100.0 & 100.0 & 97.6 & 29.3 & 39.0 & 14.6 & 24.4 & 34.1 & 97.6 & 100.0 \\
\hline Oman & 99 & 100.0 & 49.5 & 50.5 & 87.9 & 98.0 & 98.0 & 100.0 & 100.0 & 96.0 & 29.3 & 59.6 & 30.3 & 39.4 & 32.3 & 94.9 & 100.0 \\
\hline Cuba & 70 & 100.0 & 52.9 & 52.9 & 90.0 & 97.1 & 97.1 & 100.0 & 100.0 & 91.4 & 40.0 & 55.7 & 35.7 & 31.4 & 38.6 & 91.4 & 100.0 \\
\hline The Czech Republic & 125 & 100.0 & 57.0 & 59.0 & 86.0 & 94.0 & 95.0 & 100.0 & 100.0 & 94.0 & 38.0 & 52.0 & 36.0 & 26.0 & 43.0 & 92.0 & 100.0 \\
\hline Caucasians* & 465 & nt & 51.8 & 44.0 & 92.7 & nt & 93.8 & 100.0 & 100.0 & 94.0 & 41.9 & 52.0 & 26.5 & 33.6 & 40.8 & 91.2 & 100.0 \\
\hline Northern Ireland & 154 & 100.0 & 47.4 & 47.4 & 90.3 & 98.1 & 98.1 & 100.0 & 100.0 & 98.1 & 39.0 & 50.0 & 29.9 & 31.2 & 38.3 & 98.1 & 100.0 \\
\hline South Africa Xhosa & 50 & 100.0 & 64.0 & 72.0 & 64.0 & 98.0 & 96.0 & 100.0 & 100.0 & 100.0 & 4.0 & 82.0 & 38.0 & 62.0 & 10.0 & 100.0 & 100.0 \\
\hline
\end{tabular}

*Includes 147 Australians, 90 Irish, 130 Britons, 52 Americans, and another group of 40 Australians (Su et al., 2008).

\section{DISCUSSION}

KIR genes are genetically polymorphic, and are located on human chromosome 19q13.4, spanning approximately $150 \mathrm{~kb}$. They are arranged head-to-tail and clustered; their 
polymorphisms are encoded by the multi-gene family clusters, with diverse structure and function. The KIR locus contains multiple alleles, which can be expressed by the different KIR genes and vary in number and type between different ethnic groups and different areas. Different individuals have different numbers and types of KIR genes, and therefore show different haplotypes. At present, many laboratories are dedicated to KIR genotyping and haplotype composition studies, and KIR distribution profiles have been reported in more than 140 groups of the world's populations (Hsu et al., 2002; Rajalingam et al., 2002; Uhrberg et al., 2002; Jiang et al., 2005; Zhu et al., 2005; Velickovic et al., 2006; Middleton et al., 2008; Pavlova et al., 2008; Su et al., 2008; He et al., 2009; Dai et al., 2010; Wang et al., 2010).

Our study used the SSP-PCR method to interrogate the KIR gene locus in 184 individuals in the Xinjiang Han population who were the recipients or donors of a kidney transplant. We compared the test results with the other populations reported in the literature and obtained the KIR gene distribution characteristics of the Xinjiang Han population. The detection rates of 3DL3, 2DL4, 3DP1, and 3DL2 were 100\% in each group, the rates of 2DL1, 2DP1, 2DL3, and 3DL1 showed a high gene frequency of $>90 \%$; these results were consistent with the data reported from the rest of the world populations. 2DS2 and 2DL2 showed lower frequencies, similar to the published data from the Han population in Jiangsu, Shanghai, Zhejiang, and Yunnan. These were, however, significantly higher than those in the South Korean and Japan populations, and significantly lower than those in Sichuan, Hong Kong, Singaporean, Singapore, Brazil, Oman, Cuba, the Czech Republic, Caucasian, Northern Ireland, and South African Xhosa populations. The 3DS1 gene frequency of $44.0 \%$ and the 2DS1 gene frequency of $45.7 \%$ were higher than in all comparable populations. The 2DS3 gene frequency and the 2DL5 gene frequency were 23.9 and $48.4 \%$, respectively, which were higher than in the Han, Japanese, South Korean, and Caucasian populations, but significantly lower than in the Brazilian, Czech Republic, Oman, Cuban, and South African Xhosa populations. The 2DS5 gene frequency was $32.1 \%$ and significantly higher than the Han in Asia, South Korean, Singapore, and Japanese and Caucasian populations, but significantly lower than in the South African Xhosa and Oman populations. The results of this study demonstrated that the Xinjiang Han population has a unique distribution characteristic of KIR genes compared to the Han population in the other areas of China and elsewhere in Asia, as well as with the populations in other countries. This reflected that genetic background plays a large role in determining the KIR polymorphic distributions in different populations in different areas.

There were 21 KIR genotypes detected in the Xinjiang Han population, fewer than the 26 genotypes detected in the Zhejiang Han population (Zhu et al., 2005), the 30 genotypes in the Yunnan Han population (Su et al., 2008), the 34 genotypes in the Jiangsu Han population (Dai et al., 2010), and the 31 genotypes in the Sichuan Han population (Wang et al., 2010), but more than the 18 genotypes in the Shanghai Han population (Zhang et al., 2003b). Among the 21 kinds of KIR genotypes, the AJ $(2,2)$ genotype was the most common; AF $(1,2), \mathrm{R}(6$, $9), \mathrm{A}(4,4), \mathrm{AF}(1,2)$, and $\mathrm{T}(8,8)$ were less common. This profile was different than that of the AJ and AF dominating genotypes found in the Zhejiang, Shanghai, Jiangsu, and Yunnan populations (Zhu et al., 2005; Su et al., 2008; He et al., 2009), while the most common genotype in the Caucasian population was AG $(1,1)$. In addition, we found 21 genotypes in 184 Han individuals, six (from 11 individuals) of which had not been reported and could not be named in accordance with the relevant literature (Hsu et al., 2002), and were tentatively named HXJ1-6. These six new genotypes were, however, identified in the other populations in China, with IDs of $75,64,68,11,7$, and 6 . For the six genotypes, the Yunnan population IDs were 
6 and 8; Zhejiang population IDs were 11, 6, 68, 75; the Shanghai population ID was 7; and the Sichuan population ID was the same as us. The ID of 75 was the most common genotype, accounting for $2.72 \%$ in five individuals.

KIR haplotypes could be classified into two groups: haplotypes A and B. Haplotype A contained only a single activated receptor gene 2DS4, but haplotype B had more activated receptor genes. Nine haplotypes were detected in 184 Han individuals. The most common haplotype, 2 , was found in $61.3 \%$ of the subjects, followed by haplotypes 5 and 1 , which accounted for 12.7 and $8.4 \%$, respectively. Haplotypes 1 and 2 belonged to haplotype A. Haplotype A was the primary phenotype, with frequencies of $69.1 \%$ in the Xinjiang Han population. This was similar to that observed in published data from Han populations in Yunnan, Shanghai, Zhejiang, and Sichuan, with frequencies of 69.4, 74.8, 74.4, and 68.6\%. These results showed that genetic background was the major determinant for the haplotype grouping classification, regardless of where individuals from the Han population resided. It has been reported that haplotype 2 was the common haplotype in the Han populations in Yunnan (Su et al., 2008) and Shanghai (Zhang et al., 2003b), but that haplotype 1 was the common haplotype in the Caucasian population. The ratio of haplotype A was higher. It was consistent with Yunnan, Zhejiang, Shanghai, Japanese, and Korean populations. However, the major advantage was haplotype B of Australian aboriginal population, it had a difference with which the ratio of haplotype A and haplotype B of Caucasian population kept relative balance.

In this study, six new genotypes were identified for which haplotype analysis could not be performed based on Hsu's standards, suggesting that they may be new haplotypes. Zhang et al. (2003b) considered that certain special genotypes might have more than one haplotype combination. For instance, genotype A might be the combination of 1 and 10, or might also be 3 and 7; genotype $\mathrm{C}$ might be the combination of 5 and 3, or might also be 3 and 7; genotype $\mathrm{C}$ might be the combination of 5 and 3, or might also be 1 and 14. The priority in these settings was the common haplotype combination, and then was confirmed by expanding the genealogy or DNA graph.

In conclusion, the genetic polymorphism profile of the KIR locus in the Xinjiang Han population showed common characteristics of the general Han population, as well as some unique characteristics, but showed significant differences from African and Caucasian populations. China has a large population and extensive land, and represents a multi-ethnic country, with long-term coexistence of multiple populations. The KIR gene and haplotype distributions showed unique properties. At present, the theories and studies on this phenomenon are still inadequate. In order to elucidate the nature of the Chinese KIR locus, multi-regional cooperation, large sample sizes, and studies of underlying KIR structure and function should be further pursued. This study provided the basic information for further studies of KIR genes in human genetics, transplantation immunology, and genetic disease.

\section{ACKNOWLEDGMENTS}

Research supported by No. 474 People's Liberation Army Hospital, Urumqi, China, and commpled by the PCR Room Department of Blood Transfusion.

\section{REFERENCES}

Dai YD, Dong RP and Du HL (2010). Polymorphism of human killer cell immunoglobulin-like receptor gene in Chinese Jiangsu Han population. J. Exp. Hematol. 18: 776-779. 
He CT, Li L, Zhang JQ, Pan N, et al. (2009). Polymorphism of killer cell immunoglobulin receptor gene family in Han blood donors in Jiangsu Han. Chin. J. Clin. Lab. Sci. 27: 208-211.

Hsu KC, Liu XR, Selvakumar A, Mickelson E, et al. (2002). Killer Ig-like receptor haplotype analysis by gene content: evidence for genomic diversity with a minimum of six basic framework haplotype, each with multiple subsets. $J$. Immunol. 169: 5118-5129.

Jiang K, Zhu FM, Lv QF and Lan LX (2005). Distribution of killer cell immunoglobulin-like receptor genes in the Chinese Han population. Tissue Antigens 65: 556-563.

Khakoo SI and Carrington M (2006). KIR and disease: a model system of system of models? Immunol. Rev. 214: 186-201. Middleton D, Meenagh A, Moscoso J and Arnaiz-Villena A (2008). Killer immunoglobulin receptor gene and allele frequencies in Caucasoid, Oriental and Black populations from different continents. Tissue Antigens 71: 105-113.

Pavlova Y, Kolesar L, Striz I, Jabor A, et al. (2008). Distribution of KIR genes in the Czech population. Int. J. Immunogenet. 35: 57-61.

Rajalingam R, Krausa P, Shilling HG, Stein JB, et al. (2002). Distinctive KIR and HLA diversity in a panel of north Indian Hindus. Immunogenetics 53: 1009-1019.

Su PC, Yang TH, Zou H, Xu XH, et al. (2008). The Han people in Yunnan KIR gene polymorphism. Chin. J. Blood Transfus. 21: 268-272.

Uhrberg M, Parham P and Wernet P (2002). Definition of gene content for nine common group B haplotypes of the Caucasoid population: KIR haplotypes contain between seven and eleven KIR genes. Immunogenetics 54: 221-229.

Velickovic M, Velickovic Z and Dunckley H (2006). Diversity of killer cell immunoglobulin-like receptor genes in Pacific Islands populations. Immunogenetics 58: 523-532.

Wang S, Wang Y, Ji X, Yang L, et al. (2010). Polymorphism of killer cell immunoglobulin-like receptors gene in SichuanHan population, China. Chin. J. Blood Transfus. 23: 601-606.

Zhang L, Hsu KC, Liu XR, Yang YQ, et al. (2003a). Human KIR genotype PCR - SSP and family studies. Shanghai J. Immunol. 23: 99-103.

Zhang L, Hsu KC, Liu XR, Yang JQ, et al. (2003b). Killer Ig-like receptor gene content diversity and haplotype analysis in Chinese Han population in Shanghai. Chin. J. Med. Genet. 20: 396-399.

Zhu FM, Jiang K, Lv QF, Zhang W, et al. (2005). Polymorphism of killer cell immunoglobulin-like receptors gene family in Zhejiang Han population. J. Exp. Hematol. 13: 1109-1112. 